\title{
Special purpose equipment design of crystallizer copper tube for inner hole plating
}

\author{
Li Cheng ${ }^{1, a}$, Huan $\mathrm{Wu}^{2, b^{*}}$ and Xuming $\mathrm{Ye}^{3, \mathrm{c}}$ \\ ${ }^{1}$ Mechanical Engineering College, Shenyang University, Shenyang, CHN \\ ${ }^{2}$ Mechanical Engineering College, Shenyang University, Shenyang , CHN \\ ${ }^{3}$ Mechanical Engineering College, Shenyang University ,Shenyang, CHN \\ ${ }^{a} 110044,{ }^{b} 110044,{ }^{c} 110044$
}

Keywords: Continuous casting machine; Rectangular crystallizer copper tube; special purpose equipment.

Abstract. A novel special purpose equipment for inner hole plating of rectangular crystallizer copper tube in continuous casting machine was put forward. The design requirements and the main structure of the special purpose equipment were introduced. The principle and structures of the automatically centered mechanism were analyzed in detail. It is helpful to improve the efficiency and accuracy of crystallizer copper tube for inner hole plating.

\section{Introduction}

Continuous casting machine is common in iron and steel foundry industry equipment. The use of continuous casting machine greatly improves the efficiency of the cast steel. Crystallizer copper tube as the core component of continuous casting machine, its technical level determines the efficiency and the quality of the steel of cast steel. The working condition of the crystallizer copper tube is bad, due to the inner wall of the crystallizer copper tube contacts with high temperature molten steel directly for a long time. Thus, higher requirements of its material are need. Material of crystallizer copper tube are required to have not only enough high temperature strength, fatigue strength and wear resistance, low elongation and the high thermal conductivity, but also has excellent plasticity processing performance from room temperature to 400 degrees Celsius, etc. In order to prolong the service life of crystallizer copper pipe, reduce wear of copper tube wall and slab surface crack defects, and used in the crystallizer copper tube inner surface electroplating processing method, Crystallizer copper pipe inner surface is plated on a layer of protective coating .Usually chromium is chosen as plating material. Its thickness is $0.06 \sim 0.08 \mathrm{~mm}$, in order to improve the surface strength and hardness of the crystallizer copper tube inner wall.

Because of the chrome plating liquid are plating and deep plating ability is very poor, so in the crystallizer copper tube inner hole plating processing. In order to get uniformity of thickness and fine texture of cladding material, appropriate special purpose equipment must be used to ensure coaxiality requirements between crystallizer copper tube hole and positive pole. To ensure the quality of plating coating, in this case special purpose equipment need to be equipped. The current domestic special purpose equipment for inner hole plating of rectangular crystallizer copper tube, which has a heavy structure, need artificially to center positive pole and the inner hole of the crystallizer copper tube. While centering the positive pole and the crystallizer copper tube inner hole, workmen with experience usually use wedge to bit by bit operating. Artificially centering process will be affected by various subjective factors and the technical level of the workers. Thus, the whole operation process will take a long time and the precision is hard to be guar-anteed. Each batch of the alignment accuracy of the workpiece is relatively low and the alignment accuracy level is not stable. In order to ensure that each batch of workpiece can achieve the same precision, improve the work efficiency and reduce the labor intensity of workers, a kind of automatically centering of the crystallizer copper tube for inner hole plating with special purpose equipment is demanded urgently. It has become a technical problem to be solved at present. 
In this paper, a novel special purpose equipment for inner hole plating of rectangular crystallizer copper tube in continuous casting machine was put forward. The principle and structures of the automatically centered mechanism were analyzed in detail.

\section{The overall layout}

Special purpose equipment for inner hole plating of rectangular crystallizer copper tube as shown in figure 1, mainly by column, beam, suspension loop, Upper clamp plate , under clamp plate, Upper automatic centering mechanism and under automatic centering mechanism etc.
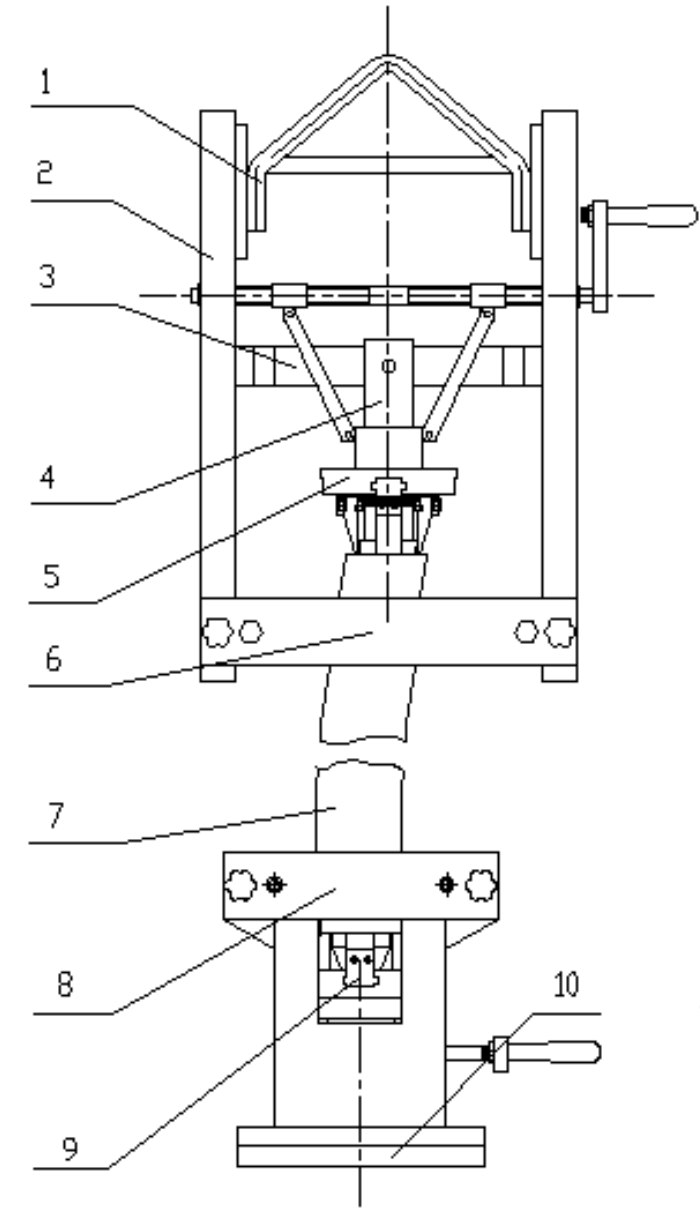

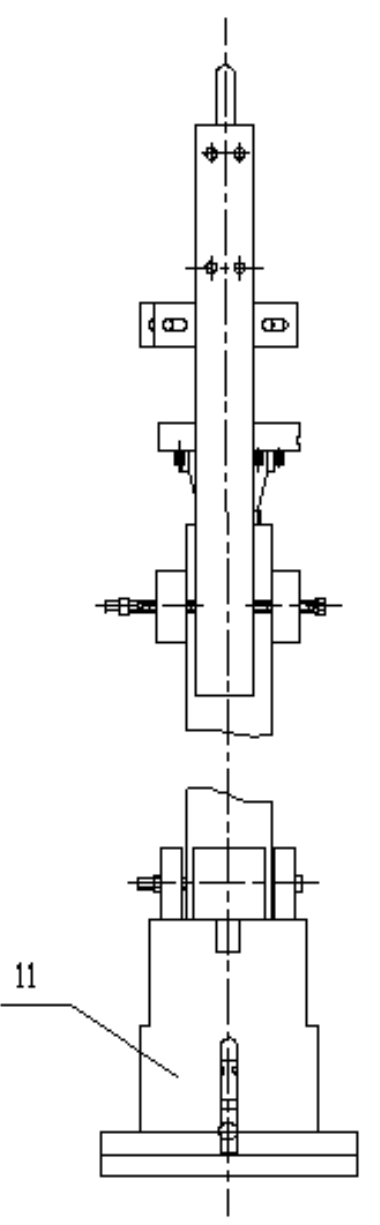

1-Suspension loop 2-Column 3-Beam 4-Positive pole 5-Upper automatic centering mechanism 6- Upper press plate 7- Crystallizer copper tube 8-Under press plate

9-Under automatic centering mechanism 10- Tapered tank column 11-Sleeve

Figure 1 Special purpose equipment for inner hole plating of rectangular crystallizer copper tube

The role of the various components are as follows:

(1)Column: used for installing the component parts of special purpose equipment for inner hole plating of rectangular crystallizer copper tube, the main supporting role.

(2)Beam: used for hanging and clamping positive pole.

(3)Suspension loop: installed on the columns, to lifting the special purpose equipment.

(4)Upper clamp plate or under clamp plate: used for clamping rectangular crystallizer copper tube.

(5)Upper automatic centering mechanism or under automatic centering mechanism: respectively used to clamp the top of the crystallizer cop-per tube and the top of positive pole, clamping the bot-tom of the crystallizer copper tube and the bottom of positive pole.

\section{General idea of automatic centering}

The hole in the crystallizer copper tube is finishing surface, the outer surface of the crystallizer copper tube is rough machining surface. Crystallizer copper tube wall thickness tolerance for wall thickness less than 5\%, the tolerance range is larger. Positioning on the outer surface of the 
crystallizer copper tube as benchmark, the benchmark misalignment error will be very big, it is difficult to achieve the requirement of alignment accuracy, so choosing rectangular crystallizer copper tube inner hole and rectangular positive pole surface as the locating basis, with four wedge as positioning components, and then together carrying out the four wedge into the four sides of the gap between crystallizer copper tube inner hole and the positive pole automatically center the crystallizer copper tube inner hole and positive pole.

One thing is important to note that the entire inside surface of the crystallizer copper tube need to be electroplated .That is to say, the entire inner hole of the crystallizer copper tube needs to be a layer of chrome plating processing. Therefore, when four wedges are as positioning components, inside surface of the crystallizer copper tube is as positioning reference, which makes crystallizer copper tube inner hole and the positive pole locate and clamp. Locating and clamping was finished, four positioning wedge must be drawn from the crystallizer copper tube inner hole. Otherwise, electroplating processing is completed, the position of the four positioning wedge retention is not plating. So when finished the locating and clamping of the crystallizer copper tube inner hole and the positive pole, the four positioning wedge must be rooted out, it is very important.

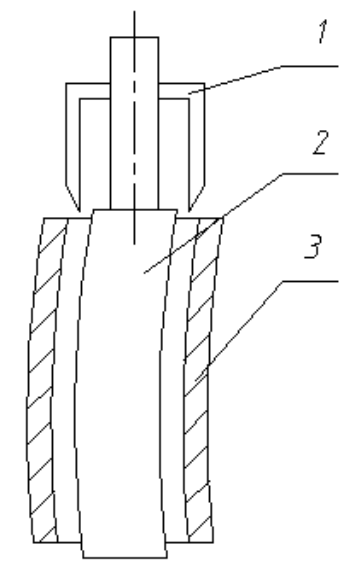

1-Wedge 2-Positive pole 3-Crystallizer copper tube

Figure 2 A process of automatic centering

As shown in figure 2, crystallizer copper tube and the positive pole in the axis direction have certain radian and a taper (crystallizer copper tube and the positive pole in the radian and taper are the same), that is, they have a pair of curved surface is parallel to each other, and the other surface is approximately parallel plane (the taper of crystallizer copper tube and the positive pole is very small, two each other with a certain angle plane can be approximately regarded as a pair of parallel plane).That is to say, along the axis direction, cross section location of crystallizer copper tube inner hole and positive pole is constantly changing, so in the process that four wedge were synchronized advancement, the radial position of them can be as the radial position of crystallizer copper tube or positive pole to the change of cross section position to constantly change, so as to avoid interference between the four wedge radial position and crystallizer copper tube or the positive pole radial position.

\section{Automatic centering mechanism}

The structure of the automatic centering mechanism as shown in figure 3 , by the handle, springs, base, sliding block, wedge. There are two kind of slideway on the base. One slideway is to cooperate with the positive pole (cooperate part is long enough, which make the base not deflect in the direction of the slideway), and the base is sliding on the positive pole; Another slideway is to cooperate with the sliding block (cooperate part is long enough, which make the sliding block not deflect in the direction of the slideway), which make the sliding block sliding on the base. Wedge is installed on the sliding block. It is driven by sliding block along the slideway of the base for radial movement, because of the crystallizer copper tube and the positive pole in the axial direction have a radian, wedge to radial movement to ensure that in the process that the four wedge is synchronized advancement or insert four sides gap between crystallizer copper tube inner hole and the positive pole, 
radial position of wedge can change along with the change of crystallizer copper tube or positive pole cross section position, avoiding interference between the four wedge radial position and crystallizer copper tube inner surface or the positive pole surface radial position. Spring force for the sliding block, which makes the wedge on the sliding block abuts on the positive pole surface. On the one hand, spring can make four wedges have always been able to insert the four sides gap between crystallizer copper tube inner hole and the positive pole. On the other hand, it is used to restrict the sliding block position and make the sliding block not be detached from the base of slideway.

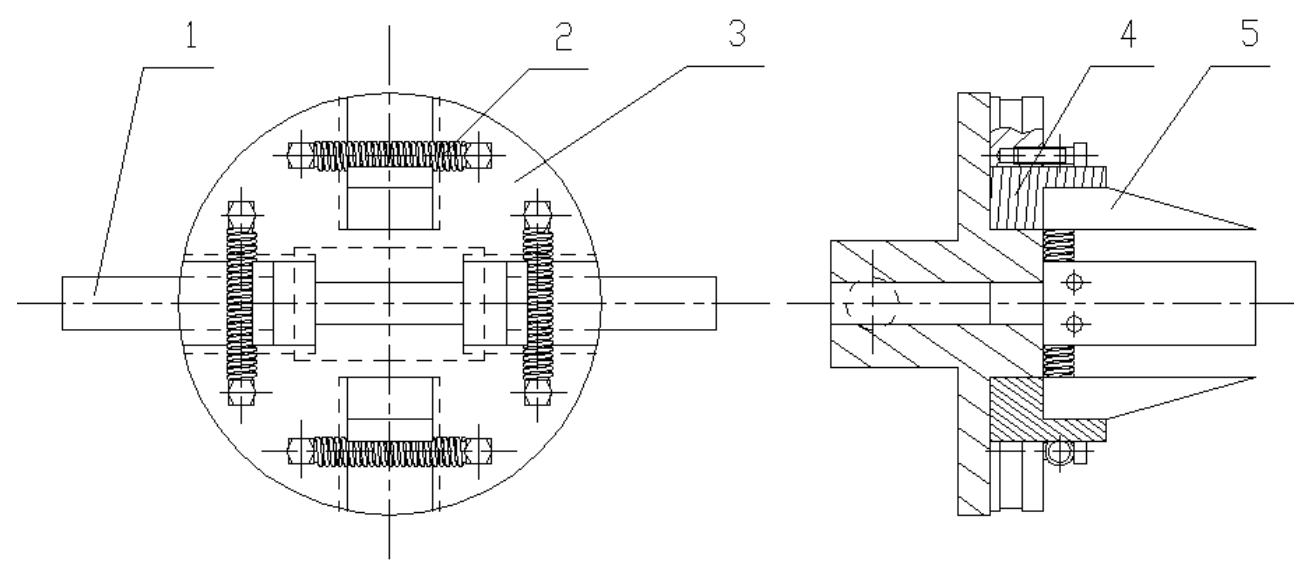

1-The handle 2-Spring 3-Base 4-Sliding block 5-Wedge

Figure 3 Automatic centering mechanism structure diagram

While pushing base, the base enables four wedge to synchronously insert to the four sides gap between crystallizer copper tube inner hole and the positive pole. Spring force make four wedge abuts on the positive pole surface, and it can take four wedge radial movement to adapt the changing of the positive pole surface radial position until four wedge swelling four sides gap between crystallizer copper tube inner hole and the positive pole surface. Thus, automatically centering of the crystallizer copper tube inner hole and positive pole can be achieved.

\section{Summary}

In this paper, a general idea that automatically centering the inner hole of rectangular crystallizer copper tube and the positive pole for plating was put forward. The principle and structures of the automatically centered mechanism were analyzed in detail. Its structure is simple and the cost is low. With this set of special purpose equipment, it is helpful to improve the working efficiency and the accuracy of crystallizer copper tube for inner hole plating, reducing the labor intensity of workers.

\section{References}

[1] Limei You, Dongjiang Peng, Jianjing Xin ed. Inner hole chrome plated fixture design and application. [J] Plating and finishing, 2013, 07:29-31.

[2] Jianchao Chen. Lug end faces in a self-centering fixture parts.[J] Mechanical workers. Cold processing, 2006, 06: 39-40.

[3] Tuo $\mathrm{Wu}$. Typical structure of modern machine tool fixture atlas. [M] Beijing: chemical industry press, 2011, 02.

[4] Dan Li, Dongxiang Shao and Jie Wang. Special machine tool fixture atlas.[M] Harbin: Harbin industrial university press, 2005, 02.

[5] Xiandong Meng. Machine tool fixture atlas.[M] Beijing: mechanical industry press, 2011, 05. 